\title{
LETTERS
}

\section{Ventilation variability inversely correlates to ejection fraction in heart failure}

\section{To the Editors:}

Heart failure is currently considered a systemic disease. In this context, there is growing interest in cardiopulmonary interactions during rest, sleep and exercise [1]. Periodic breathing during exercise is characterised by regular waxing and waning of minute ventilation $\left(V^{\prime} \mathrm{E}\right)$ due to oscillations in central respiratory drive. Periodic breathing independently predicts mortality in heart failure patients [2], but is not directly correlated with low left ventricular ejection fraction. For example, the occurrence of periodic breathing could predict cardiac mortality in patients who were waiting for cardiac transplantation, independent of ejection fraction [2].

Clinical experience shows that some heart failure patients exhibit ventilatory oscillations during exercise testing without achieving the established criteria of periodic breathing (minimum of three regular oscillations; deviation of three consecutive cycle lengths within $20 \%$ of their average; and minimal ventilatory oscillation of $5 \mathrm{~L}$ ) [2]. We hypothesised that the analysis of time-domain variability of respiratory variables during exercise could add important clinical information, detecting changes of insufficient magnitude to be classified as periodic breathing. Cardiopulmonary exercise tests in 17 heart failure patients $(53 \%$ male; mean \pm SD age $59 \pm 9$ yrs; ejection fraction $23 \pm 6 \%$ ) under standard treatment were retrospectively analysed following approval of the Research Ethics Committee of the National Institute of Traumatology and Orthopedics (Ministry of Health, Brasilia, Brazil). All maximal progressive cardiopulmonary exercise tests were performed on an electromagnetically braked cycle ergometer (Medifit 400L; Medical Fitness Equipment, Maarn, the Netherlands), with work-rate increments of $5 \mathrm{~W}$ every

\begin{tabular}{|c|c|c|c|c|c|c|}
\hline \multicolumn{2}{|c|}{ TABLE 1} & \multicolumn{5}{|c|}{$\begin{array}{l}\text { Linear correlations between ejection fraction (EF) } \\
\text { and time-domain variability of breathing variables } \\
\text { during maximal cardiopulmonary exercise } \\
\text { testing }\end{array}$} \\
\hline \multirow[t]{2}{*}{ EF } & \multicolumn{2}{|c|}{$V^{\prime} E$} & \multicolumn{2}{|r|}{ VT } & \multicolumn{2}{|r|}{$f R$} \\
\hline & $\mathrm{SD} / \mathrm{n}$ & RMSSD/n & $\mathrm{SD} / \mathbf{n}$ & RMSSD/n & $\mathrm{SD} / \mathbf{n}$ & RMSSD/n \\
\hline$r$ & -0.531 & -0.511 & -0.442 & -0.452 & -0.507 & -0.510 \\
\hline$p$-value & 0.034 & 0.043 & 0.076 & 0.068 & 0.037 & 0.036 \\
\hline
\end{tabular}

$1 \mathrm{~min}$ at $60 \mathrm{rpm}$ until exhaustion. $V^{\prime} \mathrm{E}$, respiratory frequency $(f \mathrm{R})$ and tidal volume $(V \mathrm{~T})$ were registered on a breath-bybreath basis using a computerised system (Vmax 229; SensorMedics, Buena Vista, CA, USA). The SD and root mean square successive differences (RMSSD) of $V^{\prime} \mathrm{E}, \mathrm{fR}$ and $V_{\mathrm{T}}$ during exercise testing were calculated for each patient. Considering that the number of observations has a direct influence on measures of variability, and that patients had exercised until exhaustion and thus time to exhaustion was not the same for all patients, variability (SD and RMSSD) was normalised using the number of respiratory cycles, reducing the probability that variability could be greater due to a greater number of observations registered in longer tests $(\mathrm{SD} / \mathrm{n}$ and $\mathrm{RMSSD} / \mathrm{n}$, respectively). A complete two-dimensional echocardiogram was obtained at rest. Left ventricular ejection fraction was calculated using a modified Simpson's biplane method.

Ejection fraction was inversely correlated with $V^{\prime} E$ and $f R$ variability but not with $V$ T variability (table 1 ).

Although time-domain variability of breathing variables during rest has previously been used to predict success of weaning from mechanical ventilation [3], to our knowledge, this is the first time that this analysis has been applied to exercise data from heart failure patients. The present results show that patients with lower ejection fractions exhibit greater variability in $V^{\prime} \mathrm{E}$ and $f \mathrm{R}$ throughout exercise testing. The calculations presented can be easily performed, and may add important clinical information in heart failure patients. Further studies evaluating the prognostic value of time-domain variability of $V^{\prime} \mathrm{E}$ and $f \mathrm{R}$ during exercise are warranted.

\section{R.R.T. Castro*,\#, , L.M. Antunes-Correa ${ }^{+}$, L.M. Ueno ${ }^{\S}$, M.U.P.B. Rondon ${ }^{+}$, C.E. Negrão ${ }^{+, f}$ and A.C.L. Nobrega ${ }^{\#}$}

*Exercise Physiology Laboratory, National Institute of Traumatology and Orthopedics, "Dept of Physiology and Pharmacology, Fluminense Federal University, "Medical Sciences Faculty, Rio de Janeiro State University, Rio de Janeiro, ${ }^{+}$Heart Institute, ${ }^{\S}$ School of Arts, Sciences and Humanities, and ${ }^{f}$ School of Physical Education and Sport, University of São Paulo, São Paulo, Brazil.

Correspondence: A.C.L. Nobrega, Dept of Physiology and Pharmacology, Fluminense Federal University, Rua Prof. Hernani Pires de Melo, 101, Niterói, Rio de Janeiro, 24210-130, Brazil. E-mail: aclnobrega@gmail.com

Support Statement: This study was supported by the São Paulo Research Foundation (FAPESP; São Paulo, Brazil; \#2005/ 
59740-7), National Council for Scientific and Technological Development (CNPq; Brasilia, Brazil; \#474621/2004-9), Rio de Janeiro Research Foundation (FAPERJ; Rio de Janeiro, Brazil; \#E-26/100.461/2007), and, in part, by the Zerbini Foundation (São Paulo, Brazil). L.M. Ueno was supported by FAPESP as a post-doctoral fellow (\#03/10881-2); and C.E. Negrao, M.U.P.B. Rondon and A.C.L. Nobrega were supported by $\mathrm{CNPq}$ (\#302146/2007-5, \#303518/2008-1 and \#301168/2006-7, respectively).

\section{REFERENCES}

1 Ribeiro JP. Periodic breathing in heart failure: bridging the gap between the sleep laboratory and the exercise laboratory. Circulation 2006; 113: 9-10.

2 Leite JJ, Mansur AJ, de Freitas HF, et al. Periodic breathing during incremental exercise predicts mortality in patients with chronic heart failure evaluated for cardiac transplantation. J Am Coll Cardiol 2003; 41: 2175-2181.

3 Wysocki M, Cracco C, Teixeira A, et al. Reduced breathing variability as a predictor of unsuccessful patient separation from mechanical ventilation. Crit Care Med 2006; 34: 2076-2083.

\section{Bosentan and improved pulmonary endothelial function in pulmonary arterial hypertension}

\section{To the Editors:}

Pulmonary arterial hypertension $(\mathrm{PAH})$ is characterised by pulmonary endothelial dysfunction and smooth muscle cell proliferation [1]. Miniaturised catheter-based technology has recently enabled in vivo assessment of both endothelial dysfunction and pulmonary artery thickening in vivo $[2,3]$

Bosentan, an oral dual endothelin-1 receptor antagonist, has been shown to improve clinical outcomes in PAH [4]. In vitro, bosentan has been shown to improve human endothelial cell function [5] and reduce neointimal and smooth muscle proliferation. In pigs in vivo, bosentan has been shown to partially restore hypoxia-induced reductions in nitric oxide [6]. The effect of bosentan on human pulmonary artery structure and function in vivo, however, has not been studied.

We hypothesised that bosentan therapy might improve pulmonary microvascular endothelial function and pulmonary artery remodelling in patients with established PAH. Therefore, we assessed the effect of 6 months of clinically indicated bosentan therapy in patients with advanced PAH on: 1) pulmonary microvascular function, assessed by Dopplerderived pulmonary blood flow responses to vasoactive agents; and 2) segmental pulmonary artery structure, measured by pulmonary intravascular ultrasound (IVUS).

Eight bosentan-naïve subjects (three males and five females; five subjects with idiopathic and three with sclerodermarelated PAH; mean \pm SEM age $66 \pm 4$ yrs; weight $79 \pm 5 \mathrm{~kg}$ ) were studied. The institutional review board (Royal Prince Alfred Hospital, Sydney, NSW, Australia) approved the study protocol and informed consent was obtained from all subjects. All patients were in New York Heart Association (NYHA) functional class III, had mean pulmonary arterial pressure $\left(\bar{P}_{\text {pa }}\right)>25 \mathrm{mmHg}$ at rest (without demonstrable reversibility) and had pulmonary capillary wedge pressure $\leqslant 15 \mathrm{mmHg}$.

Clinically indicated right heart catheterisation, assessing suitability for bosentan prescription, was performed. Thereafter, pulmonary artery IVUS assessment was performed in a distal segmental pulmonary artery of both upper and lower lobes of both lungs (where accessible) using Atlantis SR Pro catheters (Boston Scientific, Natick, MA, USA). Healthy sex- and agematched controls for IVUS studies were recruited from our cardiac catheterisation laboratories (Royal Prince Alfred Hospital). After documentation of normal right heart pressures in these controls, pulmonary IVUS was performed.

Next, pulmonary vascular reactivity was studied in the PAH patients, in the left lower lobe pulmonary artery, using $0.014^{\prime \prime}$ Doppler sensor guidewire (FloWire; Volcano Therapeutics, Rancho Cordova, CA, USA) connected to FloMap console (Cardiometrics, Mountain View, CA, USA), to measure Doppler flow velocity. At end-expiration, pulmonary arterial pressure, heart rate and systemic blood pressure were assessed at baseline and $2 \mathrm{~min}$ into each of the following intra-pulmonary artery drug infusions (as per our previously published methods) [7]: acetylcholine (ACh, an endothelium dependent vasodilator) to attain estimated local concentrations of $10^{-8}, 10^{-7}$ and $10^{-6} \mathrm{M}$; sodium nitroprusside (SNP, an endothelium independent vasodilator) at 7.5 and $12 \mu \mathrm{g} \cdot \mathrm{min}^{-1}$; L-nitro-mono-methyl-arginine (LNMMA, an L-arginine antimetabolite) at 2.5 and $5 \mathrm{mg} \cdot \mathrm{min}^{-1}$; and, L-arginine (a nitric oxide precursor) at $25 \mathrm{mg} \cdot \mathrm{min}^{-1}$. Selective segmental pulmonary angiograms were obtained and vessel diameters measured quantitatively offline.

Bosentan was prescribed at $62.5 \mathrm{mg}$ b.i.d. for 1 month and $125 \mathrm{mg}$ b.i.d. p.o. thereafter. At 6 months, study subjects had repeat right heart catheterisation, IVUS and microvascular reactivity testing. The selective angiograms recorded at baseline allowed correct vessel identification at follow-up.

For IVUS image analysis, the least distorted images of the most distal pulmonary artery segments of similar calibre were matched from the baseline and follow-up studies. Percentage wall thickness was calculated from total vessel lumen area measurements of 10 consecutive diastolic images, by a blinded observer.

Data are presented as mean \pm SEM. Two-way ANOVA with repeated measures was used to describe the effect of bosentan on vasoreactivity. Paired t-tests were used for other comparisons. 